\title{
Statin use and survival in patients with metastatic castration- resistant prostate cancer treated with abiraterone or enzalutamide after docetaxel failure: the international retrospective observational STABEN study
}

\author{
Jacob A. Gordon ${ }^{1, *}$, Carlo Buonerba ${ }^{2,3, *}$, Gregory Pond ${ }^{4}$, Daniel Crona5, Silke \\ Gillessen ${ }^{6}$, Giuseppe Lucarelli7, Sabrina Rossetti ${ }^{8}$, Tanya Dorff ${ }^{9}$, Salvatore Artale ${ }^{10}$, \\ Jennifer A. Locke ${ }^{1}$, Davide Bosso ${ }^{2}$, Matthew Ivan Milowsky ${ }^{5}$, Mira Sofie Witek ${ }^{6}$, \\ Michele Battaglia7, Sandro Pignata ${ }^{11}$, Cyrus Cherhroudi ${ }^{12}$, Michael E. Cox ${ }^{1}$, Pietro De \\ Placido2, Dario Ribera ${ }^{2}$, Aurelius Omlin ${ }^{6}$, Gaetano Buonocore ${ }^{13}$, Kim Chi ${ }^{14}$, Christian \\ Kollmannsberger ${ }^{14}$, Daniel Khalaf ${ }^{14}$, Gaetano Facchini ${ }^{8}$, Guru Sonpavde ${ }^{15}$, Sabino \\ De Placido², Bernhard J. Eigl ${ }^{14, \#}$ and Giuseppe Di Lorenzo ${ }^{2, \#}$

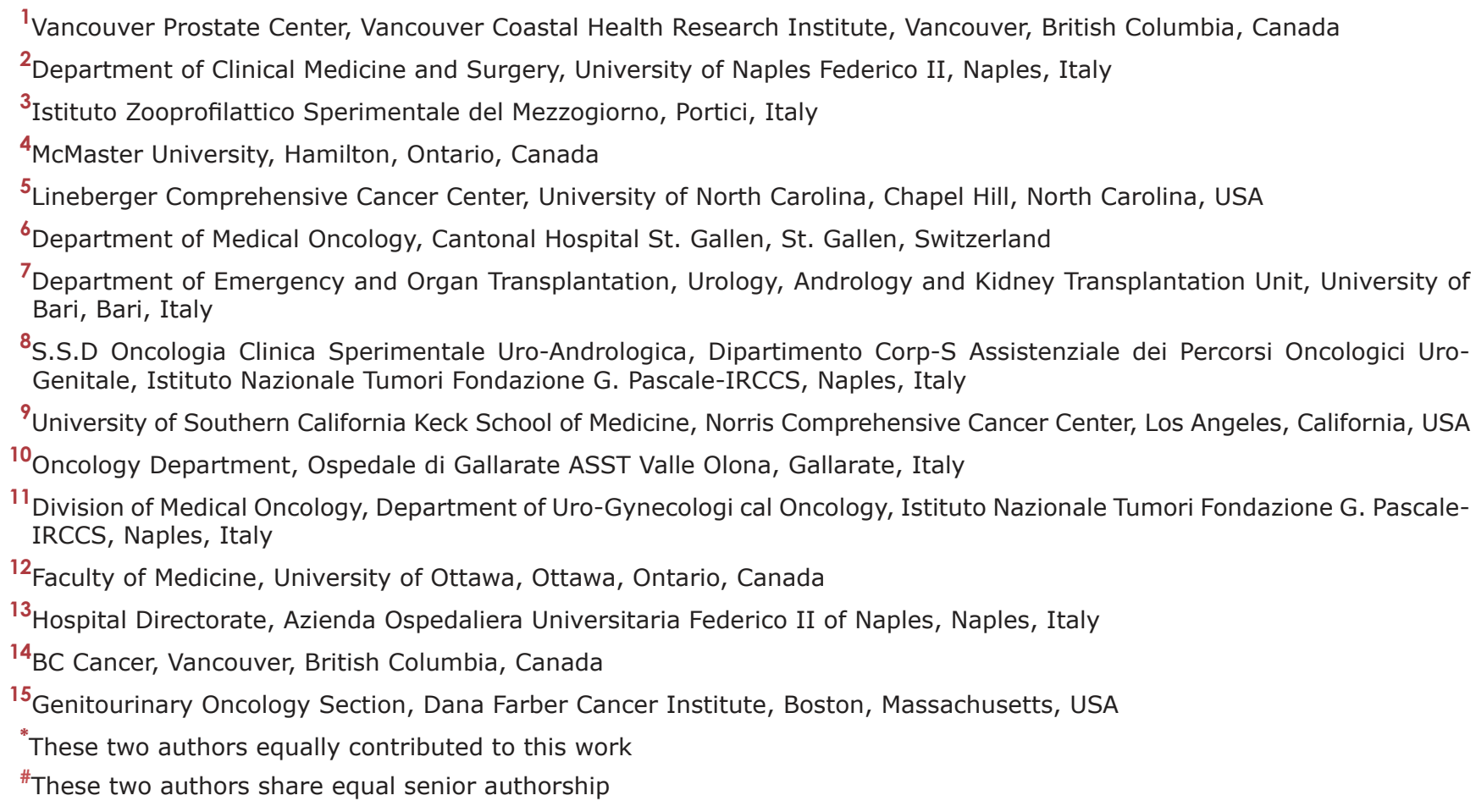 \\ Correspondence to: Giuseppe Di Lorenzo, email: dilorengiuseppe@gmail.com
}

Keywords: prostate cancer; abiraterone; enzalutamide; statins

Received: February 01, $2018 \quad$ Accepted: February 27, $2018 \quad$ Published: April 13, 2018

Copyright: Gordon et al. This is an open-access article distributed under the terms of the Creative Commons Attribution License 3.0 (CC BY 3.0), which permits unrestricted use, distribution, and reproduction in any medium, provided the original author and source are credited.

\section{ABSTRACT}

Background: Statins may potentiate the effects of anti-hormonal agents for metastatic castration-resistant prostate cancer (MCRPC) through further disruption of essential steroidogenic processes. We investigated the effects of statin use on clinical outcomes in patients with $\mathrm{MCRPC}$ receiving abiraterone or enzalutamide.

Materials and methods: This was a retrospective multicenter study including patients that received abiraterone or enzalutamide for $\mathrm{MCRPC}$. The effect of concurrent 
statin use on outcomes was evaluated. The associations of statins with early ( $\leq 12$ weeks) prostate-specific antigen (PSA) declines ( $>30 \%$ ), cancer-specific survival and overall survival (OS) were evaluated after controlling for known prognostic factors.

Results: Five hundred and ninety-eight patients treated with second-line abiraterone or enzalutamide after docetaxel for MCRPC were included. A total of 199 men $(33.3 \%)$ received statins during abiraterone/enzalutamide treatment. Median OS was 20.8 months $(95 \% \mathrm{CI}=18.3-23.2)$ for patients who received statins, versus 12.9 months $(95 \% \mathrm{CI}=11.4-14.6)$ for patients who did not receive statins $(P<$ 0.001). After adjusting for age, alkaline phosphatase, PSA, neutrophil-to-lymphocytes ratio, Charlson comorbidity score, Gleason score, visceral disease, hemoglobin, opiate use and abiraterone versus enzalutamide treatment, the use of statin therapy was associated with a $53 \%$ reduction in the overall risk of death (hazard ratio [HR] $=0.47$; $95 \% \mathrm{CI}=0.35-0.63 ; P<0.001)$. Statin use was also associated with a $63 \%$ increased odds of a $>30 \%$ PSA decline within the first 12 weeks of treatment (OR = 1.63; 95\% CI $=1.03-2.60 ; P=0.039$ ).

Conclusions: In this retrospective cohort, statin use was significantly associated with both prolonged OS and cancer-specific survival and increased early $>30 \%$ PSA declines. Prospective validation is warranted.

\section{INTRODUCTION}

In developed countries, prostate cancer is the most prevalent malignancy in men, with 142,000 patients dying each year, and an $8.8 \%$ cumulative lifetime incidence [1]. Statins are a therapeutic class of medications that are commonly prescribed to lower circulating cholesterol levels through inhibition of 3-hydroxy-3-methylglutaryl coenzyme A (HMG-CoA) reductase [2], and have an established role in primary and secondary cardiovascular prevention [3]. Over the past decade, a preponderance of evidence from numerous studies, mostly conducted in patients with hormonesensitive disease, has shown that statin use in prostate cancer patients is associated with longer cancer-specific and overall survival (OS) [4]. The putative mechanism for this observed improvement in survival is that statins may impair prostate cancer growth via multiple cholesterol- and non-cholesterol-mediated effects [4]. In a recently published study of a large, registry-based cohort, which included $>30,000$ prostate cancer patients [5], statin use was predictive of improved cancer-specific and OS, after adjusting for stage, Gleason score and primary treatment at diagnosis. Conversely, there is little evidence regarding the effects of statins among patients with castration-resistant prostate cancer (CRPC), and the potential synergism with active systemic treatments (e.g., abiraterone and enzalutamide).

Abiraterone works by inhibiting residual adrenal and intra-tumoral androgen synthesis via CYP17A blockade [6], while enzalutamide acts by inhibiting binding of testosterone to the androgen receptor (AR) as well as by blocking androgen-mediated change and nuclear translocation of AR [7]. In one small retrospective study, statin use was significantly associated with longer OS and early PSA declines in men who received abiraterone [8]. In contrast, this OS advantage has not been consistently observed in other studies $[9,10]$. Furthermore, there is prospective evidence from a phase III trial suggesting that statins may be discontinued in the palliative care setting with no detrimental effect on survival [11].

In view of the potential additive effect of statins with novel hormonal agents and of the unknown value of continuing versus discontinuing statin therapy in patients with metastatic CRPC (mCRPC), a multi-center retrospective study was conducted to further explore the effects of statin use on PSA response and survival outcomes during second-line (post-docetaxel) treatment with abiraterone or enzalutamide, after adjusting for multiple known predictive factors in the second-line setting [12].

\section{RESULTS}

\section{Patients' characteristics and outcomes}

Six hundred and forty-two patients were initially included in this dataset. Of these, 44 patients were excluded because statin use could not be ascertained. Baseline characteristics and outcomes are presented for the remaining 598 patients in Table 1A-1D. Notably, > 50\% of patients came from one treatment center (BCCA) and an additional $21 \%$ of patients came from a second center (Federico II Napoli). Median age of the population was 72 years (range, 42-96). Most of the study patients received abiraterone. Median duration of second-line treatment with abiraterone or enzalutamide was 8.3 months (range, $0.4-47.5$ ), with $52 \%$ of patients having a $>30 \%$ PSA decrease within the first 12 weeks of treatment. At the time of this analysis, $513(85.8 \%)$ patients had died, with a median OS of 16.1 months $(95 \%$ confidence interval 
Table 1A: Summary statistics

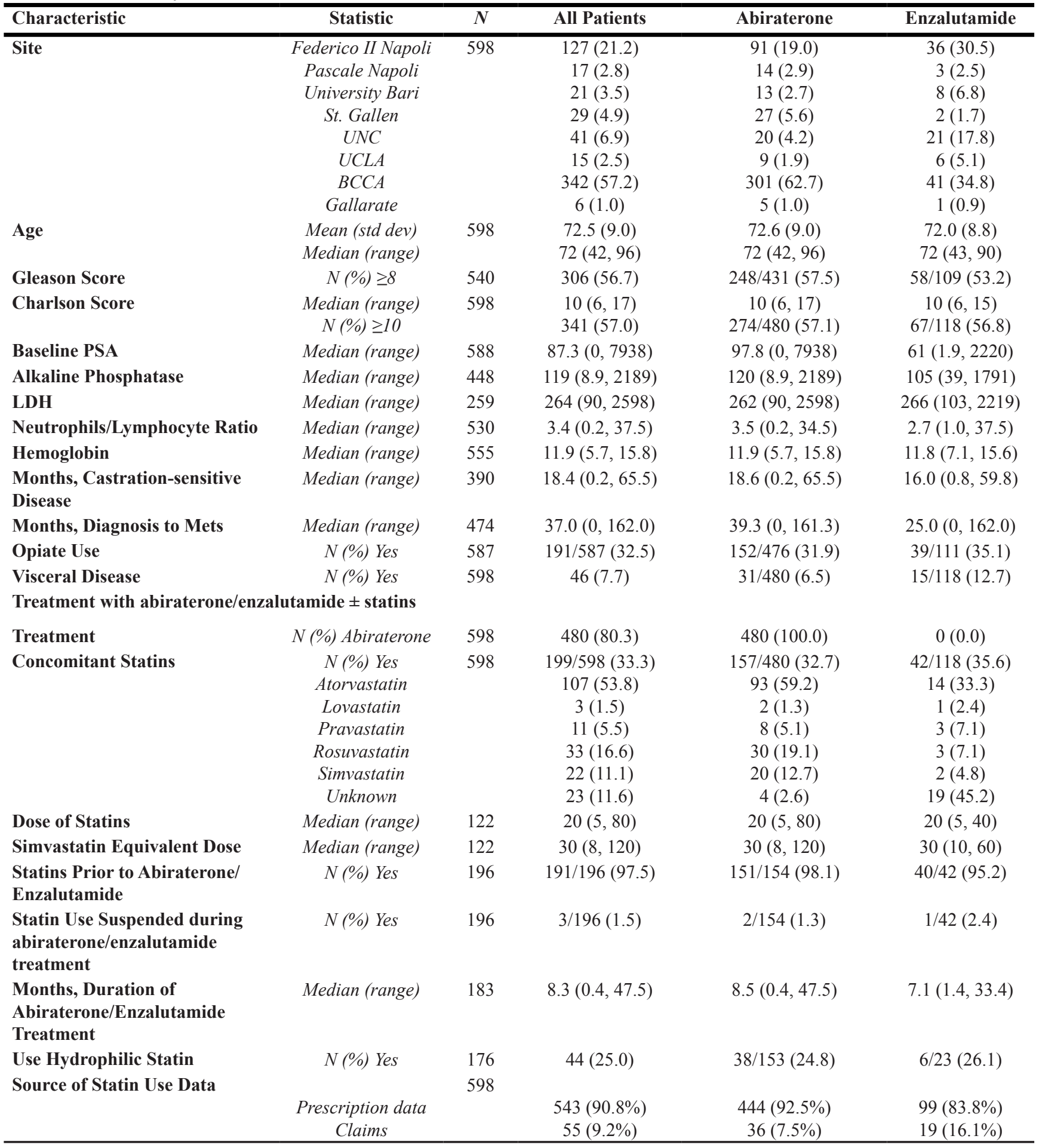

Characteristics of the study population grouped by treatment.

$[\mathrm{CI}]=13.8-17.0)$. Cancer-specific survival was 16.2 months (95\% CI: 14.3-17.1).

\section{Statin use}

Approximately one-third of the evaluable study population (199 of 598 patients) received statins during treatment, with 107 patients receiving atorvastatin (18\% of patients). Importantly, statin use was documented by the local investigator using prescription data in almost $91 \%$ of cases. Only eleven patients were reported to have started statin after abiraterone or enzalutamide or to have interrupted statins before suspending abiraterone/enzalutamide treatment ( $2 \%$ of 
Table 1B: Outcomes of the study population, grouped by treatment

\begin{tabular}{|c|c|c|c|c|c|}
\hline Characteristic & Statistic & $N$ & All Patients & Abiraterone & Enzalutamide \\
\hline \multicolumn{6}{|c|}{ Outcomes } \\
\hline$>30 \%$ PSA Decline at Week 4 & $N(\%)$ Yes & 519 & $209(40.3)$ & $169 / 419(40.3)$ & $40 / 100(40.0)$ \\
\hline$>30 \%$ PSA Decline at Week 8 & $N(\%)$ Yes & 480 & $223(46.5)$ & $184 / 391(47.1)$ & $39 / 89(43.8)$ \\
\hline$>30 \%$ PSA Decline at Week 12 & $N(\%)$ Yes & 469 & $231(49.3)$ & $184 / 383(48.0)$ & $47 / 86(54.7)$ \\
\hline $\begin{array}{l}>30 \% \text { PSA Decline at } 4,8 \text { or } 12 \\
\text { Weeks } \dagger\end{array}$ & $N(\%)$ Yes & 574 & 299/574 (52.1) & $243 / 465(52.3)$ & $56 / 109(51.4)$ \\
\hline Overall Survival & $\begin{array}{c}N(\%) \text { Deaths } \\
\text { Median }(95 \% \mathrm{CI}) \\
\text { 6-mo OS }(95 \% \mathrm{CI}) \\
\text { 1-year OS }(95 \% \mathrm{CI}) \\
\text { 2-year OS }(95 \% \mathrm{CI})\end{array}$ & 598 & $\begin{array}{l}513(85.8) \\
16.1(13.8,17.0) \\
81.7(78.3,84.6) \\
61.0(56.9,64.8) \\
31.2(27.5,35.1)\end{array}$ & $\begin{array}{l}424(88.3) \\
15.8(13.7,17.0) \\
82.4(78.7,85.6) \\
61.3(56.7,65.5) \\
30.5(26.3,34.7)\end{array}$ & $\begin{array}{l}89(75.4) \\
16.5(12.1,20.1) \\
78.5(69.9,84.9) \\
59.7(50.1,68.1) \\
34.7(25.8,43.7)\end{array}$ \\
\hline Cause of Death & Prostate Cancer & 598 & $468(91.2)$ & $390 / 424(92.0)$ & $78 / 89(87.6)$ \\
\hline Cancer-Specific Survival & $\begin{array}{l}\text { Median }(95 \% \mathrm{CI}) \\
6 \text {-mo } O S(95 \% \mathrm{CI}) \\
1 \text {-year } O S(95 \% \mathrm{CI}) \\
\text { 2-year } O S(95 \% \mathrm{CI})\end{array}$ & 598 & $\begin{array}{l}16.5(15.3,17.7) \\
82.7(79.4,85.6) \\
63.2(59.1,67.0) \\
33.8(29.8,37.8)\end{array}$ & $\begin{array}{l}16.4(14.6,17.7) \\
83.4(79.7,86.5) \\
63.3(58.7,67.5) \\
33.0(28.6,37.4)\end{array}$ & $\begin{array}{l}17.6(13.6,21.4) \\
80.0(71.4,86.2) \\
63.2(53.5,71.4) \\
37.4(28.0,46.8)\end{array}$ \\
\hline Vascular Events & $\begin{array}{c}\text { Cardiovascular N }(\%) \\
\text { Cerebrovascular N }(\%) \\
\text { Either N }(\%)\end{array}$ & 598 & $\begin{array}{l}20(3.3) \\
13(2.2) \\
33(5.5)\end{array}$ & $\begin{array}{l}15(3.1) \\
12(2.5) \\
27(5.6)\end{array}$ & $\begin{array}{l}5(4.2) \\
1(0.9) \\
6(5.1)\end{array}$ \\
\hline
\end{tabular}

$\dagger$ denominator is number of patients with a PSA assessment at week 4, 8 or 12 .

Table 1C: Summary statistics

\begin{tabular}{|c|c|c|c|c|c|}
\hline Characteristic & Statistic & $\mathbf{N}$ & No Statins & $\mathbf{N}$ & Statins \\
\hline Site & $\begin{array}{c}\text { Federico II of Napoli } \\
\text { Pascale Napoli } \\
\text { University of Bari } \\
\text { St. Gallen } \\
\text { UNC } \\
\text { UCLA } \\
\text { BCCA } \\
\text { Gallarate }\end{array}$ & 399 & $\begin{array}{l}74(18.6) \\
8(2.0) \\
14(3.5) \\
25(6.3) \\
27(6.8) \\
7(1.8) \\
241(60.4) \\
3(0.8)\end{array}$ & 199 & $\begin{array}{l}53(26.6) \\
9(4.5) \\
7(3.5) \\
4(2.0) \\
14(7.0) \\
8(4.0) \\
101(50.8) \\
3(1.5)\end{array}$ \\
\hline Age & $\begin{array}{l}\text { Mean (std dev) } \\
\text { Median (range) }\end{array}$ & 399 & $\begin{array}{l}71.9(9.4) \\
72(42,96)\end{array}$ & 199 & $\begin{array}{l}73.8(7.9) \\
74(43,94)\end{array}$ \\
\hline Gleason Score & $N(\%) \geq 8$ & 354 & $204(57.6)$ & 186 & $102(54.8)$ \\
\hline Charlson Score & $\begin{array}{c}\text { Median (range) } \\
\quad N(\%) \geq 10\end{array}$ & 399 & $\begin{array}{l}10(6,15) \\
206(51.6)\end{array}$ & 199 & $\begin{array}{l}10(6,17) \\
135(67.8)\end{array}$ \\
\hline PSA at Diagnosis & Median (range) & 391 & $95.3(0,7149)$ & 197 & $80(0.2,7938)$ \\
\hline Alkaline Phosphatase & Median (range) & 312 & $113(8.9,2189)$ & 136 & $120(25,1791)$ \\
\hline LDH & Median (range) & 175 & $260(103,2598)$ & 136 & $272(90,2219)$ \\
\hline Neutrophils/Lymphocyte Ratio & Median (range) & 358 & $3.4(0.2,34.5)$ & 172 & $3.3(0.2,37.5)$ \\
\hline Hemoglobin & Median (range) & 373 & $11.9(5.7,15.8)$ & 182 & $12.0(7.9,15.5)$ \\
\hline Months, Castration-sensitive Disease & Median (range) & 259 & $18.4(0.2,65.5)$ & 131 & $18.4(0.6,65.4)$ \\
\hline Months, Diagnosis to Metastases & Median (range) & 306 & $33.3(0,162.0)$ & 168 & $43.5(0,161.3)$ \\
\hline Opiate Use & $N(\%)$ Yes & 389 & $124(31.9)$ & 198 & $67(33.8)$ \\
\hline Visceral Disease & $N(\%) Y e s$ & 399 & $33(8.3)$ & 199 & $13(6.5)$ \\
\hline \multicolumn{6}{|l|}{ Treatment } \\
\hline Treatment & $N(\%)$ Abiraterone & 399 & $323(81.0)$ & 199 & $157(78.9)$ \\
\hline
\end{tabular}




\begin{tabular}{|c|c|c|c|c|}
\hline Concomitant Statins & $\begin{array}{l}N(\%) \text { Yes } \\
\text { Atorvastatin } \\
\text { Lovastatin } \\
\text { Pravastatin } \\
\text { Rosuvastatin } \\
\text { Simvastatin } \\
\text { Unknown }\end{array}$ & 0 & 199 & $\begin{array}{l}199(33.3) \\
107(53.8) \\
3(1.5) \\
11(5.5) \\
33(16.6) \\
22(11.1) \\
23(11.6)\end{array}$ \\
\hline Dose of Statins & Median (range) & 0 & 122 & $20(5,80)$ \\
\hline Simvastatin Equivalent Dose & Median (range) & 0 & 122 & $30(8,120)$ \\
\hline $\begin{array}{l}\text { Statins Prior to Abiraterone/ } \\
\text { Enzalutamide }\end{array}$ & $N(\%)$ Yes & 0 & 196 & $191(97.5)$ \\
\hline $\begin{array}{l}\text { Statin Use Suspended during } \\
\text { abiraterone/enzalutamide treatment }\end{array}$ & $N(\%)$ Yes & 0 & 196 & $3(1.5)$ \\
\hline $\begin{array}{l}\text { Months, Duration of Abiraterone/ } \\
\text { Enzalutamide Treatment }\end{array}$ & Median (range) & 0 & 183 & $8.3(0.4,47.5)$ \\
\hline Use of a Hydrophilic Statin & $N(\%) Y e s$ & 0 & 176 & $44(25.0)$ \\
\hline
\end{tabular}

Characteristics of the study population, grouped by statin use.

'denominator is number of patients with a PSA response assessment at week 4, 8 or 12 .

Table 1D: Outcomes of the study population, grouped by statin use

\begin{tabular}{|c|c|c|c|c|c|}
\hline Characteristic & Statistic & $N$ & No Statins & $N$ & Statins \\
\hline \multicolumn{6}{|c|}{ Outcomes } \\
\hline$>30 \%$ PSA Decline at Week 4 & $N(\%)$ Yes & 349 & $130(37.3)$ & 170 & $79(46.5)$ \\
\hline$>30 \%$ PSA Decline at Week 8 & $N(\%)$ Yes & 311 & $136(43.7)$ & 169 & $87(51.5)$ \\
\hline$>30 \%$ PSA Decline at Week 12 & $N(\%)$ Yes & 305 & $148(48.5)$ & 164 & $83(50.6)$ \\
\hline $\begin{array}{l}>30 \% \text { PSA Decline at } 4,8 \text { or } 12 \\
\text { Weeks } \dagger\end{array}$ & $N(\%)$ Yes & 380 & $186(49.0)$ & 194 & $113(58.3)$ \\
\hline Overall Survival & $\begin{array}{l}N(\%) \text { Deaths } \\
\text { Median }(95 \% \mathrm{CI}) \\
\text { 6-mo OS }(95 \% \mathrm{CI}) \\
1 \text {-year OS }(95 \% \mathrm{CI}) \\
\text { 2-year OS }(95 \% \mathrm{CI})\end{array}$ & 399 & $\begin{array}{l}347(87.0) \\
12.9(11.4,14.6) \\
78.6(74.2,82.3) \\
53.8(48.7,58.7) \\
25.9(21.6,30.5)\end{array}$ & 199 & $\begin{array}{l}166(83.4) \\
20.8(18.3,23.2) \\
87.8(82.3,91.6) \\
75.0(68.3,80.5) \\
41.6(34.5,48.4)\end{array}$ \\
\hline Cause of Death & Prostate Cancer & 347 & $324(93.4)$ & 166 & $144(86.8)$ \\
\hline Cancer-Specific Survival & $\begin{array}{l}\text { Median }(95 \% \text { CI) } \\
\text { 6-mo OS }(95 \% C I) \\
\text { 1-year OS }(95 \% C I) \\
\text { 2-year OS }(95 \% C I)\end{array}$ & 399 & $\begin{array}{l}13.4(12.1,15.8) \\
79.3(74.9,83.0) \\
56.0(50.9,60.9) \\
27.8(23.2,32.5)\end{array}$ & 199 & $\begin{array}{l}22.3(19.2,24.7) \\
89.7(84.5,93.2) \\
77.6(71.0,82.8) \\
45.5(38.1,52.6)\end{array}$ \\
\hline Vascular Events & $\begin{array}{l}\text { Cardiovascular N }(\%) \\
\text { Cerebrovascular N (\%) } \\
\text { Either N }(\%)\end{array}$ & 399 & $\begin{array}{l}8(2.0) \\
3(0.8) \\
11(2.8)\end{array}$ & 199 & $\begin{array}{l}12(6.0) \\
10(5.0) \\
22(11.1)\end{array}$ \\
\hline
\end{tabular}

patients). The median simvastatin-equivalent daily dose administered was $30 \mathrm{mg}$.

\section{Association of statins with OS and cancer-related survival}

Median OS was significantly improved for mCPRC patients who received concomitant statins, when compared to patients not treated with statins (20.8 versus
12.9 months; hazard ratio $[\mathrm{HR}]=0.57,95 \% \mathrm{CI}=0.46-$ $0.71, P<0.001$ ) (Figure 1). Table 2A summarizes the results of univariable and multivariable models for OS. In the multivariable model, statin use remained strongly associated with OS with a 53\% reduction in the risk of death. This association was similar in subgroup analyses and in the landmark analyses. Among the study patients who had died $(n=513)$, over $91 \%$ of the deaths were attributable to prostate cancer, and thus 
the cancer-specific survival was similar to OS. Median cancer-specific survival was also significantly improved for patients who received concomitant statins, when compared to patients not treated with statins (22.3 versus 13.4 months; $\mathrm{HR}=0.43,95 \% \mathrm{CI}=0.32$ to $0.58, P<$ 0.001 ) (Table 2B).

No statistically significant treatment effects were observed between enzalutamide versus abiraterone, nor were treatment differences observed based on type (atorvastatin versus other) or dose of statin.

\section{Association of statins with PSA response}

Among the 574 patients with available information, 299 (52.1\%) experienced a PSA response (>30\% decline) within 12 weeks of abiraterone or enzalutamide initiation. Early PSA responses were observed significantly more often in patients that received statins, when compared to patients who did not receive statin therapy ( $58 \%$ versus $49 \%$; odds ratio $[\mathrm{OR}]=1.46,95 \% \mathrm{CI}=1.02-2.08, P=0.04$ ) (Table 3). The association between early PSA response and statin use remained significant in the multivariable analysis $(\mathrm{OR}=1.63,95 \% \mathrm{CI}=1.03-2.60, P=0.04)$.

\section{Association of statin use and cardiovascular or cerebrovascular events}

Thirty-three study patients experienced a cardiovascular or cerebrovascular event during the time period analyzed. Timing of events was not consistently reported, and therefore time-to-event analyses could not be performed. Among the 199 patients prescribed statins, $12(6.0 \%)$ experienced a cardiovascular event, and 10 (5.0\%) experienced a cerebrovascular event. In contrast, among the 399 patients not prescribed statin therapy, 8 (2.0\%) experienced a cardiovascular event, and $3(0.8 \%)$ experienced a cerebrovascular event. After adjusting for other factors in a multivariable model, concomitant statin use remained a significant predictive factor of increased risk of cardiovascular or cerebrovascular events $(\mathrm{OR}=3.24,95 \% \mathrm{CI}=1.15-9.17, p$-value $=0.03)$ (Table 4).

\section{DISCUSSION}

Although statin use has been associated with reduced cancer-related mortality in a variety of malignancies [13], the potential synergism of statins with anti-cancer medications has been prospectively investigated only in a few clinical trials. Data from the recently published phase III double-blind, placebo-controlled LUNGSTAR trial failed to detect an OS or progression-free survival (PFS) benefit when pravastatin was added to first-line standard chemotherapy in patients with small-cell lung cancer [14]. Similarly, no benefit in overall survival associated with the use of statins added to chemotherapy was reported in two additional phase III trials conducted in advanced gastric [15] and colorectal [16] cancer patients, respectively.

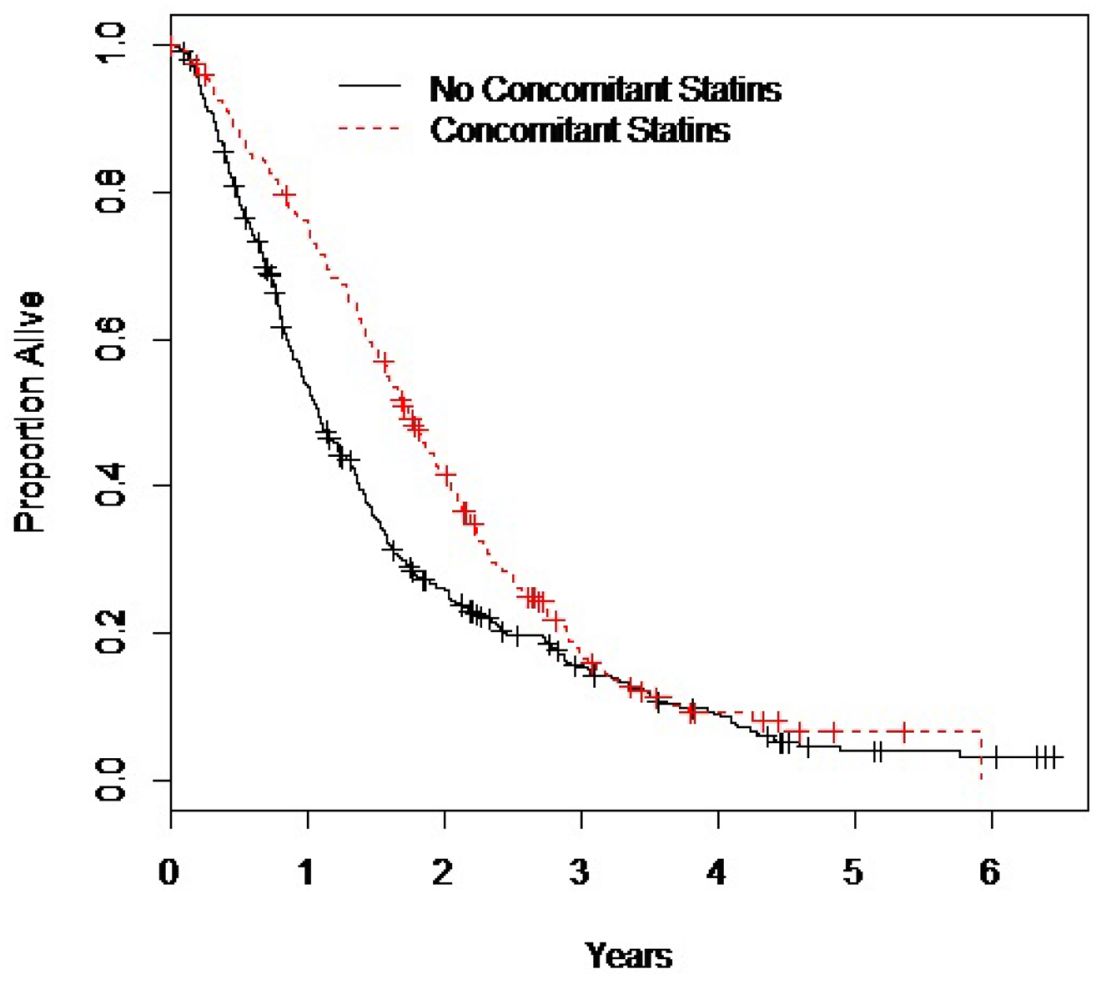

Figure 1: The Kaplan Meier curves for survival in patients receiving statins versus patients not receiving statins during abiraterone or enzalutamide treatment. 
Table 2A: Cox regression analyses, outcome $=$ overall survival

\begin{tabular}{|c|c|c|c|c|c|c|c|c|c|c|}
\hline & & \multicolumn{3}{|c|}{ All Patients } & \multicolumn{3}{|c|}{ Abiraterone } & \multicolumn{3}{|c|}{ Enzalutamide } \\
\hline & Type & $N$ & HR (95\% CI) & $P$ & $N$ & HR (95\% CI) & $P$ & $N$ & HR $(95 \%$ CI) & $P$ \\
\hline Age & / decade & 598 & $1.09(0.98,1.21)$ & 0.098 & 480 & $1.06(0.95,1.19)$ & 0.28 & 118 & $1.23(0.95,1.59)$ & 0.12 \\
\hline $\begin{array}{l}\text { Months, Castrat.- } \\
\text { Sensitive Dz }\end{array}$ & $<12 \mathrm{mos} v \mathrm{~s} \geq 12 \mathrm{mos}$ & 390 & $1.25(0.97,1.60)$ & 0.080 & 300 & $1.15(0.87,1.53)$ & 0.33 & 90 & $1.43(0.80,2.54)$ & 0.23 \\
\hline Months, Dx-Mets & $<36 \mathrm{mos} v \mathrm{~s} \geq 36 \mathrm{mos}$ & 474 & $0.94(0.77,1.16)$ & 0.57 & 370 & $0.90(0.72,1.14)$ & 0.40 & 104 & $1.03(0.65,1.65)$ & 0.89 \\
\hline Alk Phos & Log-transformed & 448 & $1.37(1.19,1.57)$ & $<0.001$ & 367 & $1.31(1.13,1.52)$ & $<0.001$ & 81 & $2.09(1.32,3.30)$ & 0.002 \\
\hline LDH & Log-transformed & 259 & $1.82(1.32,2.49)$ & $<0.001$ & 194 & $1.60(1.12,2.28)$ & 0.009 & 65 & $2.15(1.08,4.30)$ & 0.030 \\
\hline $\begin{array}{l}\text { Neutrophils/ } \\
\text { Lymphocyte Ratio }\end{array}$ & Log-transformed & 530 & $1.59(1.36,1.84)$ & $<0.001$ & 435 & $1.60(1.36,1.88)$ & $<0.001$ & 95 & $1.32(0.85,2.05)$ & 0.22 \\
\hline Hemoglobin & / unit & 555 & $0.82(0.77,0.87)$ & $<0.001$ & 450 & $0.81(0.75,0.86)$ & $<0.001$ & 105 & $0.95(0.81,1.12)$ & 0.52 \\
\hline Baseline PSA & Log-transformed & 587 & $1.21(1.14,1.29)$ & $<0.001$ & 476 & $1.20(1.13,1.28)$ & $<0.001$ & 111 & $1.27(1.07,1.51)$ & 0.007 \\
\hline Charlson Score & $\begin{array}{l}\text { / unit } \\
\geq 10 \text { vs }<10\end{array}$ & 598 & $\begin{array}{l}1.03(0.99,1.08) \\
1.10(0.92,1.32)\end{array}$ & $\begin{array}{l}0.17 \\
0.29\end{array}$ & 480 & $\begin{array}{l}1.03(0.98,1.08) \\
1.13(0.92,1.38)\end{array}$ & $\begin{array}{l}0.28 \\
0.25\end{array}$ & 118 & $\begin{array}{l}1.03(0.90,1.17) \\
0.91(0.58,1.43)\end{array}$ & $\begin{array}{l}0.69 \\
0.69\end{array}$ \\
\hline Gleason Score & $\geq 8 v s<8$ & 540 & $1.16(0.96,1.42)$ & 0.13 & 431 & $1.18(0.94,1.48)$ & 0.15 & 109 & $0.99(0.63,1.57)$ & 0.98 \\
\hline Visceral Disease & Yes vs No & 598 & $1.67(1.18,2.35)$ & 0.004 & 480 & $1.59(1.05,2.40)$ & 0.028 & 118 & $1.71(0.89,3.30)$ & 0.11 \\
\hline Opiates & Yes vs No & 587 & $0.98(0.80,1.21)$ & 0.85 & 476 & $1.07(0.86,1.34)$ & 0.52 & 111 & $0.52(0.29,0.94)$ & 0.030 \\
\hline Treatment & Enza vs Abi & 598 & $1.10(0.87,1.40)$ & 0.42 & & - & & & - & \\
\hline Concomitant Statins & Yes vs No & 598 & $0.57(0.46,0.71)$ & $<0.001$ & 480 & $0.58(0.45,0.73)$ & $<0.001$ & 118 & $0.61(0.37,1.01)$ & 0.052 \\
\hline Statin Type & Atorvastatin vs Other & 199 & $1.05(0.75,1.48)$ & 0.77 & 157 & $1.21(0.82,1.77)$ & 0.34 & 42 & $0.41(0.12,1.38)$ & 0.15 \\
\hline $\begin{array}{l}\text { Simvastatin } \\
\text { Equivalent Dose }\end{array}$ & $/ m g$ & 123 & $1.00(0.99,1.01)$ & 0.51 & 100 & $0.99(0.98,1.01)$ & 0.28 & 23 & $1.03(0.97,1.08)$ & 0.33 \\
\hline $\begin{array}{l}\text { Use of a hydrophilic } \\
\text { statin }\end{array}$ & Yes vs No & 176 & $0.78(0.52,1.16)$ & 0.22 & 153 & $0.64(0.42,0.98)$ & 0.040 & 23 & $2.29(0.63,8.29)$ & 0.21 \\
\hline \multicolumn{11}{|c|}{ Multivariable Model } \\
\hline Age & / decade & 387 & $1.10(0.94,1.29)$ & 0.25 & 319 & $1.09(0.92,1.30)$ & 0.33 & 68 & $1.30(0.86,1.98)$ & 0.21 \\
\hline Alk Phos & Log-transformed & & $1.24(1.06,1.46)$ & 0.008 & & $1.21(1.02,1.43)$ & 0.031 & & $2.98(1.60,5.58)$ & $<0.001$ \\
\hline $\begin{array}{l}\text { Neutrophils/ } \\
\text { Lymphocyte Ratio }\end{array}$ & Log-transformed & & $1.56(1.30,1.89)$ & $<0.001$ & & $1.63(1.33,2.00)$ & $<0.001$ & & $0.93(0.49,1.76)$ & 0.82 \\
\hline Hemoglobin & / unit & & $0.85(0.78,0.93)$ & $<0.001$ & & $0.83(0.76,0.91)$ & $<0.001$ & & $1.04(0.81,1.35)$ & 0.75 \\
\hline Baseline PSA & Log-transformed & & $1.13(1.05,1.22)$ & 0.002 & & $1.13(1.04,1.22)$ & 0.003 & & $1.35(1.02,1.79)$ & 0.038 \\
\hline Charlson Score & $\geq 10 v s<10$ & & $0.95(0.72,1.24)$ & 0.69 & & $0.96(0.71,1.30)$ & 0.81 & & $0.79(0.38,1.65)$ & 0.53 \\
\hline Gleason Score & $\geq 8 v s<8$ & & $1.13(0.88,1.45)$ & 0.34 & & $1.16(0.88,1.54)$ & 0.29 & & $1.52(0.76,3.03)$ & 0.24 \\
\hline Visceral Disease & Yes vs No & & $1.93(1.24,3.01)$ & 0.004 & & $1.81(1.08,3.03)$ & 0.025 & & $3.25(1.11,9.53)$ & 0.032 \\
\hline Opiate Use & Yes vs No & & $1.10(0.85,1.42)$ & 0.48 & & $1.28(0.97,1.68)$ & 0.080 & & $0.22(0.09,0.57)$ & 0.002 \\
\hline Treatment & Enza vs Abi & & $0.91(0.66,1.25)$ & 0.56 & & - & - & & - & - \\
\hline Concomitant Statins & Yes vs No & & $0.47(0.35,0.63)$ & $<0.001$ & & $0.45(0.33,0.62)$ & $<0.001$ & & $0.41(0.19,0.92)$ & 0.031 \\
\hline \multicolumn{11}{|c|}{ 3-Month Landmark Analysis - Multivariable Model. } \\
\hline Age & / decade & 360 & $1.07(0.91,1.27)$ & 0.41 & 296 & $1.06(0.88,1.27)$ & 0.54 & 64 & $1.19(0.77,1.84)$ & 0.43 \\
\hline Alk Phos & Log-transformed & & $1.15(0.97,1.36)$ & 0.12 & & $1.11(0.92,1.33)$ & 0.27 & & $3.13(1.63,6.02)$ & $<0.001$ \\
\hline $\begin{array}{l}\text { Neutrophils/ } \\
\text { Lymphocyte Ratio }\end{array}$ & Log-transformed & & $1.51(1.23,1.85)$ & $<0.001$ & & $1.59(1.27,1.97)$ & $<0.001$ & & $0.93(0.47,1.83)$ & 0.83 \\
\hline Hemoglobin & /unit & & $0.86(0.78,0.94)$ & 0.001 & & $0.85(0.77,0.93)$ & 0.001 & & $1.00(0.75,1.34)$ & 0.99 \\
\hline Baseline PSA & Log-transformed & & $1.13(1.05,1.22)$ & 0.002 & & $1.13(1.04,1.22)$ & 0.004 & & $1.33(0.97,1.84)$ & 0.078 \\
\hline Charlson Score & $\geq 10 v s<10$ & & $0.93(0.70,1.22)$ & 0.59 & & $0.97(0.71,1.33)$ & 0.86 & & $0.75(0.34,1.62)$ & 0.46 \\
\hline Gleason Score & $\geq 8 v s<8$ & & $1.16(0.89,1.51)$ & 0.26 & & $1.25(0.93,1.69)$ & 0.14 & & $1.17(0.56,2.43)$ & 0.67 \\
\hline Visceral Disease & Yes vs No & & $2.05(1.28,3.30)$ & 0.003 & & $1.79(1.02,3.13)$ & 0.043 & & $3.61(1.12,10.86)$ & 0.023 \\
\hline Opiates & Yes vs No & & $1.16(0.89,1.51)$ & 0.28 & & $1.27(0.96,1.69)$ & 0.099 & & $0.30(0.11,0.79)$ & 0.015 \\
\hline Treatment & Enza vs $A b i$ & & $0.86(0.62,1.20)$ & 0.38 & & - & - & & - & - \\
\hline $\begin{array}{l}\geq 3 \text { Months of } \\
\text { Continuous Use } \\
\text { Statins }\end{array}$ & Yes vs No & & $0.53(0.39,0.72)$ & $<0.001$ & & $0.51(0.37,0.72)$ & $<0.001$ & & $0.49(0.20,1.24)$ & 0.13 \\
\hline
\end{tabular}


Table 2B: Cox regression analyses, outcome = cancer-specific survival

\begin{tabular}{|c|c|c|c|c|c|c|c|c|c|c|}
\hline & & \multicolumn{3}{|c|}{ All Patients } & \multicolumn{3}{|c|}{ Abiraterone Only } & \multicolumn{3}{|c|}{ Enzalutamide Only } \\
\hline & Type & $N$ & HR (95\% CI) & $P$ & $N$ & HR (95\% CI) & $P$ & $N$ & HR (95\% CI) & $P$ \\
\hline Age & / decade & 598 & $1.03(0.93,1.15)$ & 0.60 & 480 & $1.01(0.90,1.13)$ & 0.93 & 118 & $1.16(0.88,1.54)$ & 0.29 \\
\hline $\begin{array}{l}\text { Months, Castrat.- } \\
\text { Sensitive Dz }\end{array}$ & $<12 \mathrm{mos} v \mathrm{~s} \geq 12 \mathrm{mos}$ & 390 & $1.26(0.98,1.63)$ & 0.073 & 300 & $1.18(0.88,1.57)$ & 0.27 & 90 & $1.35(0.74,2.46)$ & 0.33 \\
\hline Months, Dx-Mets & $<36 \mathrm{mos} v \mathrm{~s} \geq 36 \mathrm{mos}$ & 474 & $0.90(0.72,1.11)$ & 0.32 & 370 & $0.87(0.69,1.12)$ & 0.28 & 104 & $0.97(0.59,1.60)$ & 0.90 \\
\hline Alk Phos & Log-transformed & 448 & $1.38(1.19,1.59)$ & $<0.001$ & 367 & $1.32(1.13,1.54)$ & $<0.001$ & 81 & $2.34(1.44,3.80)$ & $<0.001$ \\
\hline LDH & Log-transformed & 259 & $1.94(1.41,2.69)$ & $<0.001$ & 194 & $1.68(1.17,2.42)$ & 0.005 & 65 & $2.50(1.24,5.03)$ & 0.010 \\
\hline $\begin{array}{l}\text { Neutrophils/ } \\
\text { Lymphocyte Ratio }\end{array}$ & Log-transformed & 530 & $1.70(1.45,1.99)$ & $<0.001$ & 435 & $1.70(1.43,2.02)$ & $<0.001$ & 95 & $1.37(0.85,2.21)$ & 0.20 \\
\hline Hemoglobin & / unit & 555 & $0.80(0.75,0.86)$ & $<0.001$ & 450 & $0.79(0.74,0.85)$ & $<0.001$ & 105 & $0.92(0.78,1.10)$ & 0.36 \\
\hline Baseline PSA & Log-transformed & 587 & $1.23(1.16,1.31)$ & $<0.001$ & 476 & $1.22(1.14,1.30)$ & $<0.001$ & 111 & $1.29(1.07,1.56)$ & 0.008 \\
\hline Charlson Score & $\begin{array}{l}\text { /unit } \\
\geq 10 \mathrm{vs}<10\end{array}$ & 598 & $\begin{array}{l}1.00(0.96,1.06) \\
1.02(0.84,1.22)\end{array}$ & $\begin{array}{l}0.88 \\
0.88\end{array}$ & 480 & $\begin{array}{l}1.00(0.94,1.05) \\
1.04(0.84,1.27)\end{array}$ & $\begin{array}{l}0.89 \\
0.74\end{array}$ & 118 & $\begin{array}{l}1.01(0.88,1.15) \\
0.79(0.48,1.29)\end{array}$ & $\begin{array}{l}0.94 \\
0.34\end{array}$ \\
\hline Gleason Score & $\geq 8 v s<8$ & 540 & $1.21(0.98,1.48)$ & 0.077 & 431 & $1.20(0.95,1.52)$ & 0.13 & 109 & $1.05(0.64,1.72)$ & 0.85 \\
\hline Visceral Disease & Yes vs No & 598 & $1.66(1.16,2.36)$ & 0.005 & 480 & $1.52(0.99,2.33)$ & 0.056 & 118 & $1.86(0.95,3.62)$ & 0.069 \\
\hline Opiates & Yes vs No & 587 & $1.01(0.82,1.26)$ & 0.91 & 476 & $1.08(0.86,1.36)$ & 0.51 & 111 & $0.59(0.32,1.09)$ & 0.094 \\
\hline Treatment & Enza vs Abi & 598 & $1.04(0.81,1.34)$ & 0.77 & & - & & & - & \\
\hline Concomitant Statins & Yes vs No & 598 & $0.51(0.41,0.64)$ & $<0.001$ & 480 & $0.52(0.40,0.67)$ & $<0.001$ & 118 & $0.53(0.31,0.93)$ & 0.025 \\
\hline Statin Type & Atorvastatin vs Other & 199 & $1.04(0.72,1.51)$ & 0.84 & 157 & $1.17(0.78,1.78)$ & 0.45 & 42 & $0.41(0.12,1.38)$ & 0.15 \\
\hline $\begin{array}{l}\text { Simvastatin } \\
\text { Equivalent Dose }\end{array}$ & $/ m g$ & 123 & $1.00(0.99,1.01)$ & 0.47 & 100 & $0.99(0.98,1.01)$ & 0.26 & 23 & $1.03(0.97,1.08)$ & 0.33 \\
\hline $\begin{array}{l}\text { Use of a hydrophilic } \\
\text { statin }\end{array}$ & Yes vs No & 176 & $0.77(0.50,1.18)$ & 0.24 & 153 & $0.62(0.39,0.98)$ & 0.043 & 23 & $2.29(0.63,8.29)$ & 0.21 \\
\hline \multicolumn{11}{|c|}{ Multivariable Model } \\
\hline Age & / decade & 387 & $1.03(0.87,1.21)$ & 0.77 & 319 & $1.03(0.86,1.23)$ & 0.79 & 68 & $1.25(0.79,1.99)$ & 0.34 \\
\hline Alk Phos & Log-transformed & & $1.21(1.02,1.43)$ & 0.026 & & $1.18(0.98,1.40)$ & 0.075 & & $3.91(1.88,8.10)$ & $<0.001$ \\
\hline $\begin{array}{l}\text { Neutrophils/ } \\
\text { Lymphocyte Ratio }\end{array}$ & Log-transformed & & $1.62(1.33,1.98)$ & $<0.001$ & & $1.66(1.35,2.06)$ & $<0.001$ & & $0.98(0.48,2.00)$ & 0.95 \\
\hline Hemoglobin & / unit & & $0.84(0.77,0.92)$ & $<0.001$ & & $0.83(0.75,0.91)$ & $<0.001$ & & $0.99(0.76,1.30)$ & 0.97 \\
\hline Baseline PSA & Log-transformed & & $1.16(1.07,1.26)$ & $<0.001$ & & $1.15(1.06,1.25)$ & $<0.001$ & & $1.44(1.05,1.98)$ & 0.024 \\
\hline Charlson Score & $\geq 10 v s<10$ & & $0.93(0.70,1.23)$ & 0.61 & & $0.95(0.70,1.30)$ & 0.74 & & $0.63(0.29,1.38)$ & 0.25 \\
\hline Gleason Score & $\geq 8 v s<8$ & & $1.13(0.87,1.47)$ & 0.35 & & $1.15(0.85,1.54)$ & 0.36 & & $1.76(0.82,3.78)$ & 0.15 \\
\hline Visceral Disease & Yes vs No & & $1.87(1.17,2.97)$ & 0.008 & & $1.68(0.97,2.90)$ & 0.063 & & $4.17(1.34,12.96)$ & 0.013 \\
\hline Opiates & Yes vs No & & $1.15(0.88,1.51)$ & 0.31 & & $1.29(0.97,1.72)$ & 0.080 & & $0.19(0.07,0.52)$ & 0.001 \\
\hline Treatment & Enza vs $A b i$ & & $0.87(0.62,1.22)$ & 0.41 & & - & - & & - & - \\
\hline Concomitant Statins & Yes vs No & & $0.43(0.32,0.58)$ & $<0.001$ & & $0.41(0.29,0.57)$ & $<0.001$ & & $0.37(0.16,0.87)$ & 0.023 \\
\hline \multicolumn{11}{|c|}{ 3-Month Landmark Analysis - Multivariable Model } \\
\hline Age & / decade & 360 & $0.98(0.83,1.17)$ & 0.86 & 296 & $0.98(0.81,1.19)$ & 0.87 & 64 & $1.05(0.66,1.70)$ & 0.83 \\
\hline Alk Phos & Log-transformed & & $1.10(0.92,1.32)$ & 0.29 & & $1.06(0.88,1.29)$ & 0.52 & & $4.00(1.87,8.58)$ & $<0.001$ \\
\hline $\begin{array}{l}\text { Neutrophils/ } \\
\text { Lymphocyte Ratio }\end{array}$ & Log-transformed & & $1.56(1.26,1.94)$ & $<0.001$ & & $1.62(1.28,2.03)$ & $<0.001$ & & $0.97(0.46,2.06)$ & 0.94 \\
\hline Hemoglobin & / unit & & $0.85(0.77,0.94)$ & 0.001 & & $0.84(0.76,0.93)$ & 0.001 & & $0.98(0.73,1.33)$ & 0.91 \\
\hline Baseline PSA & Log-transformed & & $1.16(1.07,1.26)$ & $<0.001$ & & $1.15(1.06,1.26)$ & 0.001 & & $1.34(0.95,1.90)$ & 0.096 \\
\hline Charlson Score & $\geq 10$ vs $<10$ & & $0.92(0.69,1.23)$ & 0.56 & & $0.96(0.69,1.32)$ & 0.78 & & $0.64(0.28,1.45)$ & 0.28 \\
\hline Gleason Score & $\geq 8 v s<8$ & & $1.17(0.89,1.54)$ & 0.27 & & $1.25(0.91,1.71)$ & 0.17 & & $1.17(0.53,2.58)$ & 0.70 \\
\hline Visceral Disease & Yes vs No & & $1.97(1.19,3.23)$ & 0.008 & & $1.64(0.90,3.00)$ & 0.10 & & $4.21(1.36,13.07)$ & 0.013 \\
\hline Opiates & Yes vs No & & $1.20(0.91,1.58)$ & 0.21 & & $1.28(0.95,1.72)$ & 0.10 & & $0.27(0.10,0.77)$ & 0.015 \\
\hline Treatment & Enza vs $A b i$ & & $0.84(0.59,1.20)$ & 0.33 & & - & - & & - & - \\
\hline $\begin{array}{l}\geq 3 \text { Months of } \\
\text { Continuous Use Statins }\end{array}$ & Yes vs No & & $0.48(0.35,0.66)$ & $<0.001$ & & $0.47(0.33,0.66)$ & $<0.001$ & & $0.46(0.17,1.22)$ & 0.12 \\
\hline
\end{tabular}


Table 3: Logistic regression analyses, outcome $=$ early $30 \%$ PSA decline

\begin{tabular}{|c|c|c|c|c|c|c|c|c|c|c|}
\hline & & \multicolumn{3}{|c|}{ All Patients } & \multicolumn{3}{|c|}{ Abiraterone } & \multicolumn{3}{|c|}{ Enzalutamide } \\
\hline & Type & $N$ & OR $(95 \%$ CI $)$ & $P$ & $N$ & OR $(95 \%$ CI $)$ & $P$ & $N$ & OR $(95 \%$ CI $)$ & $P$ \\
\hline Age & /decade & 574 & $1.05(0.87,1.27)$ & 0.63 & 465 & $1.20(0.96,1.48)$ & 0.10 & 109 & $0.60(0.35,1.02)$ & 0.059 \\
\hline $\begin{array}{l}\text { Months, Castration- } \\
\text { sensitive Disease }\end{array}$ & $<12 \mathrm{mos} v \mathrm{~s} \geq 12 \mathrm{mos}$ & 376 & $0.77(0.49,1.22)$ & 0.27 & 293 & $0.69(0.41,1.17)$ & 0.17 & 83 & $1.07(0.40,2.92)$ & 0.89 \\
\hline $\begin{array}{l}\text { Months, Disease- } \\
\text { Metastases }\end{array}$ & $<36 \mathrm{mos} v \mathrm{~s} \geq 36 \mathrm{mos}$ & 457 & $0.72(0.49,1.05)$ & 0.085 & 360 & $0.62(0.40,0.96)$ & 0.031 & 97 & $1.09(0.46,2.54)$ & 0.85 \\
\hline Alk Phos & Log-transformed & 433 & $1.10(0.85,1.41)$ & 0.49 & 355 & $1.18(0.89,1.56)$ & 0.26 & 78 & $0.74(0.39,1.37)$ & 0.33 \\
\hline LDH & Log-transformed & 255 & $0.72(0.41,1.26)$ & 0.24 & 192 & $0.91(0.47,1.75)$ & 0.78 & 63 & $0.44(0.14,1.42)$ & 0.17 \\
\hline $\begin{array}{l}\text { Neutrophils/ } \\
\text { Lymphocyte Ratio }\end{array}$ & Log-transformed & 516 & $0.99(0.76,1.28)$ & 0.91 & 423 & $0.97(0.73,1.30)$ & 0.86 & 93 & $1.46(0.68,3.15)$ & 0.34 \\
\hline Hemoglobin & lunit & 540 & $1.17(1.04,1.32)$ & 0.008 & 438 & $1.16(1.01,1.32)$ & 0.034 & 102 & $1.15(0.90,1.46)$ & 0.27 \\
\hline Baseline PSA & Log-transformed & 572 & $1.02(0.91,1.14)$ & 0.75 & 464 & $1.03(0.92,1.17)$ & 0.60 & 108 & $1.00(0.76,1.32)$ & 0.99 \\
\hline Charlson Score & $\begin{array}{c}\text { /unit } \\
\geq 10 \mathrm{vs}<10\end{array}$ & 574 & $\begin{array}{l}1.02(0.94,1.12) \\
1.07(0.76,1.50)\end{array}$ & $\begin{array}{l}0.62 \\
0.71\end{array}$ & 465 & $\begin{array}{l}1.06(0.96,1.17) \\
1.36(0.93,1.99)\end{array}$ & $\begin{array}{l}0.23 \\
0.11\end{array}$ & 109 & $\begin{array}{l}0.86(0.67,1.10) \\
0.43(0.19,1.00)\end{array}$ & $\begin{array}{c}0.22 \\
0.051\end{array}$ \\
\hline Gleason Score & $\geq 8 v s<8$ & 520 & $0.58(0.40,0.85)$ & 0.005 & 419 & $0.54(0.35,0.83)$ & 0.005 & 101 & $0.88(0.39,2.01)$ & 0.76 \\
\hline Visceral Disease & Yes vs No & 574 & $0.52(0.27,1.00)$ & 0.050 & 465 & $0.71(0.33,1.52)$ & 0.38 & 109 & $0.32(0.09,1.09)$ & 0.068 \\
\hline Opiate Use & Yes vs No & 571 & $0.92(0.62,1.37)$ & 0.69 & 463 & $0.93(0.60,1.44)$ & 0.74 & 108 & $1.04(0.38,2.82)$ & 0.94 \\
\hline Treatment & $\begin{array}{l}\text { Enzalutamide vs } \\
\text { Abiraterone }\end{array}$ & 574 & $0.95(0.61,1.47)$ & 0.81 & & - & & & - & \\
\hline Concomitant Statins & Yes vs No & 574 & $1.46(1.02,2.08)$ & 0.040 & 465 & $1.57(1.05,2.34)$ & 0.030 & 109 & $1.09(0.48,2.48)$ & 0.85 \\
\hline Statin Type & Atorvastatin vs Other & 194 & $0.76(0.40,1.42)$ & 0.38 & 154 & $0.77(0.38,1.58)$ & 0.48 & 40 & $0.59(0.10,3.59)$ & 0.56 \\
\hline Dose of Statins & $/ m g$ & 122 & $1.00(0.98,1.01)$ & 0.60 & 99 & $1.00(0.98,1.02)$ & 0.93 & 23 & $0.92(0.84,1.02)$ & 0.11 \\
\hline $\begin{array}{l}\text { Use of a hydrophilic } \\
\text { statin }\end{array}$ & Yes vs No & 173 & $1.06(0.52,2.16)$ & 0.88 & 150 & $1.18(0.55,2.55)$ & 0.67 & 23 & $0.76(0.10,5.94)$ & 0.80 \\
\hline \multicolumn{11}{|c|}{ Multivariable Model } \\
\hline Age & /decade & 379 & $0.98(0.72,1.33)$ & 0.87 & 312 & $1.04(0.74,1.47)$ & 0.83 & 67 & $0.76(0.35,1.64)$ & 0.48 \\
\hline Alk Phos & Log-transformed & & $1.06(0.78,1.44)$ & 0.70 & & $1.17(0.84,1.64)$ & 0.36 & & $0.51(0.20,1.31)$ & 0.16 \\
\hline $\begin{array}{l}\text { Neutrophils/ } \\
\text { Lymphocyte Ratio }\end{array}$ & Log-transformed & & $1.16(0.83,1.62)$ & 0.37 & & $1.15(0.80,1.65)$ & 0.46 & & $1.93(0.62,6.01)$ & 0.26 \\
\hline Hemoglobin & /unit & & $1.22(1.04,1.44)$ & 0.015 & & $1.21(1.01,1.45)$ & 0.043 & & $1.11(0.76,1.62)$ & 0.60 \\
\hline Baseline PSA & Log-transformed & & $1.09(0.93,1.26)$ & 0.29 & & $1.06(0.90,1.25)$ & 0.50 & & $1.26(0.78,2.02)$ & 0.35 \\
\hline Charlson Score & $\geq 10 v s<10$ & & $1.07(0.65,1.77)$ & 0.80 & & $1.31(0.74,2.31)$ & 0.36 & & $0.40(0.11,1.42)$ & 0.16 \\
\hline Gleason Score & $\geq 8 v s<8$ & & $0.69(0.43,1.10)$ & 0.12 & & $0.73(0.43,1.23)$ & 0.24 & & $0.80(0.26,2.50)$ & 0.70 \\
\hline Visceral Disease & Yes vs No & & $0.66(0.28,1.53)$ & 0.33 & & $0.74(0.26,2.08)$ & 0.57 & & $0.51(0.11,2.41)$ & 0.40 \\
\hline Opiates & Yes vs No & & $0.97(0.59,1.57)$ & 0.89 & & $1.09(0.64,1.87)$ & 0.75 & & $0.77(0.19,3.20)$ & 0.72 \\
\hline Treatment & Enza vs $A b i$ & & $1.45(0.81,2.60)$ & 0.21 & & - & - & & - & - \\
\hline Concomitant Statins & Yes vs No & & $1.63(1.03,2.60)$ & 0.039 & & $1.80(1.06,3.06)$ & 0.029 & & $1.02(0.32,3.21)$ & 0.97 \\
\hline
\end{tabular}

Biologically, statins can potentiate the efficacy of anti-androgen treatments, such as abiraterone and enzalutamide, in mCRPC through a number of potential mechanisms, including: inhibition of intra-tumoral de novo steroid biosynthesis [17], inhibition of biosynthesis of isoprenoids [18], as well as inhibition of the organic anionic transporters (e.g., SLCO2B1) [19] that are responsible for adrenal androgen dehydroepiandrosterone (DHEA) influx into cancer cells [20].

In one translational study, Harshman et al. [21] showed that statins impaired DHEA influx through competitive inhibition of the SLCO2B1 transporter both in both androgen-dependent ( $\mathrm{LNCaP})$ and partially androgen-dependent (22RV1) prostate cancer cell lines. This was supported by their retrospective clinical study of 926 patients, treated with androgen deprivation, which demonstrated that patients who received statin therapy experienced longer median time to progression, when compared to patients not treated with a statin (27.5 versus 17.4 months; $P<0.001)$. Because abiraterone is also a SLCO2B1 substrate, the same research group [10] hypothesized that statin use could be a negative predictive factor for patients taking abiraterone. However, their retrospective study of 224 abiraterone-treated patients demonstrated that statin use trended toward longer treatment duration (14.2 versus 9.2 months; HR: 0.79, 
Table 4: Logistic regression analyses of cardiovascular or cerebrovascular events

\begin{tabular}{|c|c|c|c|c|}
\hline & & \multicolumn{3}{|c|}{ All Patients } \\
\hline & Type & $N$ & OR $(95 \% \mathrm{CI})$ & $\boldsymbol{P}$ \\
\hline Age & /decade & 598 & $2.24(1.46,3.46)$ & $<0.001$ \\
\hline Months, Castration-sensitive Disease & $<12 \mathrm{mos} v \mathrm{~s} \geq 12 \mathrm{mos}$ & 390 & $0.55(0.16,1.97)$ & 0.36 \\
\hline Months, Disease-Metastases & $<36 \mathrm{mos} v \mathrm{~s} \geq 36 \mathrm{mos}$ & 474 & $1.14(0.51,2.55)$ & 0.75 \\
\hline Alk Phos & Log-transformed & 448 & $0.94(0.54,1.64)$ & 0.83 \\
\hline LDH & Log-transformed & 259 & $0.89(0.26,3.03)$ & 0.85 \\
\hline Neutrophils/Lymphocyte Ratio & Log-transformed & 530 & $1.38(0.81,2.36)$ & 0.24 \\
\hline Hemoglobin & /unit & 555 & $1.02(0.80,1.31)$ & 0.85 \\
\hline PSA at Diagnosis & Log-transformed & 587 & $0.94(0.74,1.18)$ & 0.57 \\
\hline Charlson Score & $\begin{array}{c}\text { /unit } \\
\geq 10 \text { vs }<10\end{array}$ & 598 & $\begin{array}{l}1.54(1.29,1.84) \\
4.51(1.72,11.85)\end{array}$ & $\begin{array}{c}<0.001 \\
0.002\end{array}$ \\
\hline Gleason Score & $\geq 8 v s<8$ & 540 & $0.57(0.27,1.19)$ & 0.13 \\
\hline Visceral Disease & Yes vs No & 598 & $0.36(0.05,2.71)$ & 0.32 \\
\hline Opiate Use & Yes vs No & 587 & $0.68(0.30,1.54)$ & 0.35 \\
\hline Treatment & Enzalutamide vs Abiraterone & 598 & $0.90(0.36,2.23)$ & 0.82 \\
\hline Concomitant Statins & Yes vs No & 598 & $4.38(2.08,9.24)$ & $<0.001$ \\
\hline Statin Type & Atorvastatin vs Other & 199 & $1.58(0.63,3.96)$ & 0.33 \\
\hline Dose of Statins & $/ m g$ & 123 & $1.02(0.99,1.04)$ & 0.22 \\
\hline Use of a hydrophilic statin & Yes vs No & 176 & $0.73(0.23,2.30)$ & 0.58 \\
\hline \multicolumn{5}{|c|}{ Multivariable Analysis } \\
\hline Age & /decade & 387 & $2.56(1.11,5.89)$ & 0.028 \\
\hline Alk Phos & Log-transformed & & $1.39(0.61,3.19)$ & 0.43 \\
\hline Neutrophils/Lymphocyte Ratio & Log-transformed & & $1.24(0.52,2.94)$ & 0.63 \\
\hline Hemoglobin & /unit & & $1.11(0.73,1.70)$ & 0.62 \\
\hline PSA at Diagnosis & Log-transformed & & $0.65(0.45,0.93)$ & 0.020 \\
\hline Charlson Score & $\geq 10 v s<10$ & & $1.56(0.43,5.70)$ & 0.50 \\
\hline Gleason Score & $\geq 8 v s<8$ & & $0.77(0.24,2.46)$ & 0.66 \\
\hline Visceral Disease & Yes vs No & & $0.64(0.07,6.28)$ & 0.70 \\
\hline OpiateUse & Yes vs No & & $0.72(0.22,2.38)$ & 0.59 \\
\hline Treatment & Enzalutamide vs Abiraterone & & $0.58(0.12,2.78)$ & 0.50 \\
\hline Concomitant Statins & Yes vs No & & $3.24(1.15,9.17)$ & 0.027 \\
\hline
\end{tabular}

95\% CI, 0.57-1.09, $P=0.14)$. Despite lack of validation in an independent cohort of 270 abiraterone-treated patients [10], the authors concluded that concomitant stain use did not negatively impact survival.

In our previous retrospective observational study ( $n=187$ mCRPC patients from 10 participating centers who received abiraterone), statin use was associated with longer $\mathrm{OS}$ in univariate $(\mathrm{HR}=0.51,95 \% \mathrm{CI}=0.37-0.72$, $P<0.001)$ and multivariate analyses $(\mathrm{HR}=0.40$, $95 \% \mathrm{CI}=0.27-0.59, P<0.001)$. Statin use was also significantly associated with early PSA declines $(>50 \%$ declines at week 12 in statin users versus non-users: $72.1 \%$ vs. $38.5 ; P<0.001)$. This study was limited by several factors, including the relatively small sample size, the lack of information about statin type and statin treatment duration, comorbidities, cardiovascular events, and prostate cancer-specific survival. To overcome these limitations, we designed a retrospective observational study to be conducted in an international setting that could better define concomitant treatment with statins. One of the purposes of the STABEN trial was to assess whether the potential advantage associated with statin use could be related to their known cardiovascular and cerebrovascular protective effect, of particular potential importance in an elderly population receiving abiraterone - an agent with known cardiovascular toxicity [22]. In the present retrospective study, multivariable models that included known prognostic factors in prostate cancer (e.g., baseline 
PSA levels, hemoglobin levels, Gleason score, alkaline phosphatase and LDH levels [23], visceral involvement [24] and neutrophil to lymphocyte ratio [25]) revealed that statin use was associated with a $53 \%$ reduction in the risk of all-cause mortality, and a $57 \%$ reduction in the risk of prostate cancer-specific mortality. It also appeared that statin co-administration increased the odds of having an early $>30 \%$ PSA decrease, which is consistent with our previously reported findings and adds strength to the hypothesis of a potential synergism with abiraterone/ enzalutamide.

Notably, the positive effect of statins on survival did not appear to be influenced by the known protective statin effect against vascular events. While the observed incidence of cardiovascular events reported in this study are consistent with previously reported rates of grade 2 or higher abiraterone-associated cardiovascular events [22], mCRPC patients from this study who were prescribed statin therapy appeared to be at an approximately 4-fold greater risk of experiencing a vascular event. Although such analyses did not account for time-toevent, competing risks, or a history of pre-existing cardiac conditions, this finding could be explained by the observation that patients prescribed statins often present with a greater number of co-morbidities and therefore a greater cardio- and cerebro-vascular risk, when compared to non-statin users [26]. Furthermore, the protective effect of statins was maintained after correcting for Charlson comorbidity index. Although statin consumption was modeled by using a binary variable, it must be noted that only a few patients were not prescribed statins throughout the entire abiraterone or enzalutamide treatment duration, which does not make useful to model statin exposure as a time-dependent variable. Finally, the novelty of the STABEN study also relies in the increased survival in mCRPC patients receiving concomitant enzalutamide and statins vs. enzalutamide alone, which is consistent with the multiple putative pharmacodynamic interactions of statins with anti-androgen receptor agents.

Despite its larger sample size versus published series [8-10], this study still suffers from the limitations that apply to retrospective studies, including the lack of data on some key factors such as LDH and time from castration-sensitive disease, as well as the non-systematic selection of participating centers.

\section{CONCLUSIONS}

In the large retrospective, observational STABEN study, we found a positive association of statin use with overall- and cancer specific- survival in patients receiving abiraterone or enzalutamide in the secondline setting after docetaxel failure. Statin use was documented by using high-quality prescription data in most patients. The positive association found in our patient cohort with survival was reported both in abiraterone- and enzalutamide-treated men and was consistent with early $>30 \%$ PSA declines. Analyzed together with previous epidemiology and biological findings, the STABEN results may serve as the basis to design prospective clinical trials assessing the value of adding statins to abiraterone or enzalutamide in mCRPC patients. Optimizing statin use in patients with advanced prostate cancer represents a compelling clinical opportunity to improve survival via the addition of a safe and inexpensive drug.

\section{MATERIALS AND METHODS}

\section{Inclusion criteria}

Medical records were reviewed at eight participating centers for patients with diagnosed mCRPC who were treated with second-line abiraterone or enzalutamide between January 2011 and January 2016. Histologicallyconfirmed prostate cancer and previous docetaxel-based treatment were required for inclusion in this study. Castration-resistance was determined per Prostate Cancer Clinical Trials Working Group 2 (PCWG2) criteria [22]. Patients who received at least one 28-day cycle of abiraterone or enzalutamide in the second-line setting were regarded as eligible for this study. Patient data including medical and prostate cancer history, demographic, and baseline characteristics were retrieved starting at the time of abiraterone or enzalutamide initiation. Data collected regarding statin use included: type and dose of statin prescribed, source of the data (claims versus prescription data), and dates of statin use initiation and discontinuation.

\section{Data analysis}

Summary statistics were used to describe patient outcomes. Time-to-event outcomes were calculated from the first date of treatment with abiraterone or enzalutamide.

The primary objective of this study was to determine whether concomitant statin therapy was predictive of OS improvement for mCRPC patients treated with secondline abiraterone or enzalutamide. The secondary objective of the study was to determine whether concomitant statin therapy was predictive of early ( $\leq 12$ weeks $)>30 \%$ PSA declines. The Kaplan-Meier method was used to estimate differences in survival between mCRPC patients treated who did and did not receive statin therapy, while Cox proportional hazards regression was used to investigate prognostic factors of overall survival. Logistic regression was used to investigate predictive factors of early $>30 \%$ PSA declines. Using Cox proportional hazards, multivariable models were constructed to examine the effects of concomitant statins after adjusting for all other potential sources of variation. However, there were large numbers of missing data for some factors. Thus, a priori, 
it was decided to include only those factors which had $<30 \%$ missing data and were significant on univariate analysis, or those factors with $<15 \%$ missing data overall. The impact of statins was then assessed after adjusting for factors included in the multivariable model. Supportive analyses were performed by including only those treated with abiraterone ( $\sim 80 \%$ of the cohort), only those treated with enzalutamide, by performing a cancer-specific survival analysis and by performing a landmark analysis using 3-months as the landmark time. For the purposes of the landmark analysis, any patient who was not prescribed statin therapy at the time of abiraterone or enzalutamide initiation, experienced interruption of statin therapy, or received less than 3 months of statin therapy, was deemed to not have received statins. Data modifications were performed for statistical purposes. Specifically, a logarithmic transformation was used on covariates which were highly non-normal. Duration from prostate cancer diagnosis to detection of metastases, and duration of prostate cancer diagnosis to determination of castrationresistant disease were dichotomized. All analyses included site as a stratification factor. All tests were two-sided and a $p$-value of 0.05 or less was considered statistically significant. No $p$-value adjustments were performed due to multiple testing; however, inferences were performed understanding that multiple analyses were performed.

\section{ACKNOWLEDGMENTS AND FUNDING}

The authors would like to acknowledge contribution by Isabella Ricci from the Gallarate Hospital; by Giuseppe Bassi, Antonio Verde, Francesca Vitrone, Luigi Marone, Simona Iaccarino, Mirta Mosca, Simona De Vivo, Francesco Amaniera, Antonella Mollo, Salvatore Cipolla and Chiara Rapolla from University Federico II of Naples; by Thomas De los Reyes from the Vancouver Cancer Center.

The study was partially supported by LILT sez. Napoli and Benevento.

\section{CONFLICTS OF INTEREST}

Omlin Aurelius: AO Advisory role (compensated, institutional): Astra Zeneca, Astellas, Bayer, Janssen, MSD, Pfizer, Roche, Sanofi Aventis. Research support (institutional): Teva, Janssen. Travel suport: Astellas, Bayer, Sanofi Aventis

Silke GIllesen: Speaker bureau (uncompensat ed,institutional):Astellas, Roche and Sanofi; Speaker bureau (compensated, institutional): Ferring, Janseen, Novartis; Consultant (compensated, institutional): AAA International; Astellas; Bayer; Bristol-Myers Squibb; Clovis; CureVac ; Ferring ; Janssen ; MaxiVax SA ; Roche ; Sanofi ; Consultant (uncompensated, institutional): ESSA Pharmaceuticals ; Nectar ; ProteoMediX

Guru Sonpavde: Consultant for Bayer, Sanofi, Pfizer, Novartis, Eisai, Janssen, Amgen, Astrazeneca, Merck,
Genentech, Argos, Agensys, EMD Serono; Research support to institution from Bayer, Amgen-Onyx, Celgene, Boehringer-Ingelheim, Merck, Pfizer; Author for Uptodate; Speaker for Clinical Care Options, PER (Physicians Education Resource), RTP (Research To Practice), Onclive

Bernhard J. Eigl: Consultant for Janssen; Travel support for conferences from Janssen

Carlo Buonerba: Consultant for Sanofi; Travel support from Pfizer, Janssen, Sanofi; Research support to institution from Quercegen, Astellas, Sanofi

Giuseppe Di Lorenzo: Speaker bureau: Sanofi, Bristol-Meier-Squibb, Astellas, Janssen, Pfizer; Consultant for Sanofi; Research support to institution from Quercegen, Astellas, Sanofi

Tanya Dorff: Consultant for Janssen disclose.

All other authors declare they have nothing to

\section{REFERENCES}

1. Torre LA, Bray F, Siegel RL, Ferlay J, Lortet-Tieulent J, Jemal A. Global cancer statistics, 2012. CA Cancer J Clin. 2015; 65:87-108. https://doi.org/10.3322/caac.21262.

2. Gu Q, Paulose-Ram R, Burt VL, Kit BK. Prescription cholesterol-lowering medication use in adults aged 40 and over: United States, 2003-2012. NCHS Data Brief. 2014; 177:1-8.

3. Naci H, Brugts JJ, Fleurence R, Tsoi B, Toor H, Ades AE. Comparative benefits of statins in the primary and secondary prevention of major coronary events and all-cause mortality: a network meta-analysis of placebo-controlled and activecomparator trials. Eur J Prev Cardiol. 2013; 20:641-57. https://doi.org/10.1177/2047487313480435.

4. Alfaqih MA, Allott EH, Hamilton RJ, Freeman MR, Freedland SJ. The current evidence on statin use and prostate cancer prevention: are we there yet? Nat Rev Urol. 2017; 14:107-19. https://doi.org/10.1038/nrurol.2016.199.

5. Larsen SB, Dehlendorff C, Skriver C, Dalton SO, Jespersen CG, Borre M, Brasso K, Norgaard M, Johansen C, Sorensen HT, Hallas J, Friis S. Postdiagnosis Statin Use and Mortality in Danish Patients With Prostate Cancer. J Clin Oncol. 2017; 35:3290-7. https://doi.org/10.1200/ JCO.2016.71.8981.

6. Grist E, Attard G. The development of abiraterone acetate for castration-resistant prostate cancer. Urol Oncol. 2015; 33:289-94. https://doi.org/10.1016/j.urolonc.2015.03.021.

7. Bhattacharya S, Hirmand $M$, Phung $D$, van Os $S$. Development of enzalutamide for metastatic castrationresistant prostate cancer. Ann N Y Acad Sci. 2015; 1358:13-27. https://doi.org/10.1111/nyas.12846.

8. Di Lorenzo G, Sonpavde G, Pond G, Lucarelli G, Rossetti S, Facchini G, Scagliarini S, Carteni G, Federico P, Daniele B, Morelli F, Bellelli T, Ferro M, et al. Statin Use and Survival in Patien ts with Metastatic Castration-resistant Prostate Cancer Treated with Abiraterone Acetate. Eur Urol Focus. 
2017 Apr 8. https://doi.org/10.1016/j.euf.2017.03.015. [Epub ahead of print].

9. Boegemann M, Schlack K, Fischer AK, Gerss J, Steinestel J, Semjonow A, Schrader AJ, Krabbe LM. Influence of Statins on Survival Outcome in Patients with Metastatic Castration Resistant Prostate Cancer Treated with Abiraterone Acetate. PLoS One. 2016; 11:e0161959. https://doi.org/10.1371/ journal.pone.0161959.

10. Harshman LC, Werner L, Tripathi A, Wang X, Maughan BL, Antonarakis ES, Nakabayashi M, McKay R, Pomerantz M, Mucci LA, Taplin ME, Sweeney CJ, Lee GM, et al. The impact of statin use on the efficacy of abiraterone acetate in patients with castration-resistant prostate cancer. Prostate. 2017; 77:1303-11. https://doi.org/10.1002/pros.23390.

11. Kutner JS, Blatchford PJ, Taylor DH Jr, Ritchie CS, Bull JH, Fairclough DL, Hanson LC, LeBlanc TW, Samsa GP, Wolf S, Aziz NM, Currow DC, Ferrell B, et al. Safety and benefit of discontinuing statin therapy in the setting of advanced, life-limiting illness: a randomized clinical trial. JAMA Intern Med. 2015; 175:691-700. https://doi.org/10.1001/ jamainternmed.2015.0289.

12. Buonerba C, Pond GR, Sonpavde G, Federico P, Rescigno P, Puglia L, Bosso D, Virtuoso A, Policastro T, Izzo M, Vaccaro L, Ferro M, Aieta M, et al. Potential value of Gleason score in predicting the benefit of cabazitaxel in metastatic castration-resistant prostate cancer. Future Oncol. 2013; 9:889-97. https://doi.org/10.2217/fon.13.39.

13. Zhong S, Zhang X, Chen L, Ma T, Tang J, Zhao J. Statin use and mortality in cancer patients: Systematic review and meta-analysis of observational studies. Cancer Treat Rev. 2015; 41:554-67. https://doi.org/10.1016/j.ctrv.2015.04.005.

14. Seckl MJ, Ottensmeier CH, Cullen M, Schmid P, Ngai Y, Muthukumar D, Thompson J, Harden S, Middleton G, Fife KM, Crosse B, Taylor P, Nash S, et al. Multicenter, Phase III, Randomized, Double-Blind, Placebo-Controlled Trial of Pravastatin Added to First-Line Standard Chemotherapy in Small-Cell Lung Cancer (LUNGSTAR). J Clin Oncol. 2017; 35:1506-14. https://doi.org/10.1200/JCO.2016.69.7391.

15. Kim ST, Kang JH, Lee J, Park SH, Park JO, Park YS, Lim HY, Hwang IG, Lee SC, Park KW, Lee HR, Kang WK. Simvastatin plus capecitabine-cisplatin versus placebo plus capecitabine-cisplatin in patients with previously untreated advanced gastric cancer: a double-blind randomised phase 3 study. Eur J Cancer. 2014; 50:2822-30. https://doi. org/10.1016/j.ejca.2014.08.005.

16. Lim SH, Kim TW, Hong YS, Han SW, Lee KH, Kang HJ, Hwang IG, Lee JY, Kim HS, Kim ST, Lee J, Park JO, Park SH, et al. A randomised, double-blind, placebocontrolled multi-centre phase III trial of XELIRI/FOLFIRI plus simvastatin for patients with metastatic colorectal cancer. Br J Cancer. 2015; 113:1421-6. https://doi.org/10.1038/ bjc.2015.371.

17. Gordon JA, Midha A, Szeitz A, Ghaffari M, Adomat HH, Guo Y, Klassen TL, Guns ES, Wasan KM, Cox ME. Oral simvastatin administration delays castration-resistant progression and reduces intratumoral steroidogenesis of LNCaP prostate cancer xenografts. Prostate Cancer Prostatic Dis. 2016; 19:21-7. https://doi.org/10.1038/pcan.2015.37.

18. Roy M, Kung HJ, Ghosh PM. Statins and prostate cancer: role of cholesterol inhibition vs. prevention of small GTPbinding proteins. Am J Cancer Res. 2011; 1:542-61.

19. Noe J, Portmann R, Brun ME, Funk C. Substrate-dependent drug-drug interactions between gemfibrozil, fluvastatin and other organic anion-transporting peptide (OATP) substrates on OATP1B1, OATP2B1, and OATP1B3. Drug Metab Dispos. 2007; 35:1308-14. https://doi.org/10.1124/ dmd.106.012930.

20. Wright JL, Kwon EM, Ostrander EA, Montgomery RB, Lin DW, Vessella R, Stanford JL, Mostaghel EA. Expression of SLCO transport genes in castration-resistant prostate cancer and impact of genetic variation in SLCO1B3 and SLCO2B1 on prostate cancer outcomes. Cancer Epidemiol Biomarkers Prev. 2011; 20:619-27. https://doi. org/10.1158/1055-9965.EPI-10-1023.

21. Harshman LC, Wang X, Nakabayashi M, Xie W, Valenca L, Werner L, Yu Y, Kantoff AM, Sweeney CJ, Mucci LA, Pomerantz M, Lee GS, Kantoff PW. Statin Use at the Time of Initiation of Androgen Deprivation Therapy and Time to Progression in Patients With Hormone-Sensitive Prostate Cancer. JAMA Oncol. 2015; 1:495-504. https:// doi.org/10.1001/jamaoncol.2015.0829.

22. Moreira RB, Debiasi M, Francini E, Nuzzo PV, Velasco G, Maluf FC, Fay AP, Bellmunt J, Choueiri TK, Schutz FA. Differential side effects profile in patients with $\mathrm{mCRPC}$ treated with abiraterone or enzalutamide: a meta-analysis of randomized controlled trials. Oncotarget. 2017; 8:84572-8. https://doi.org/10.18632/oncotarget.20028.

23. Miller K, Carles J, Gschwend JE, Van Poppel H, Diels J, Brookman-May SD. The Phase 3 COU-AA-302 Study of Abiraterone Acetate Plus Prednisone in Men with Chemotherapy-naive Metastatic Castration-resistant Prostate Cancer: Stratified Analysis Based on Pain, Prostate-specific Antigen, and Gleason Score. Eur Urol. 2017 Sep 20. https:/ / doi.org/10.1016/j.eururo.2017.08.035. [Epub ahead of print].

24. Gandaglia G, Karakiewicz PI, Briganti A, Passoni NM, Schiffmann J, Trudeau V, Graefen M, Montorsi F, Sun M. Impact of the Site of Metastases on Survival in Patients with Metastatic Prostate Cancer. Eur Urol. 2015; 68:325-34. https://doi.org/10.1016/j.eururo.2014.07.020.

25. van Soest RJ, Efstathiou JA, Sternberg CN, Tombal B. The Natural History and Outcome Predictors of Metastatic Castration-resistant Prostate Cancer. Eur Urol Focus. 2016; 2:480-7. https://doi.org/10.1016/j.euf.2016.12.006.

26. Thomsen RW, Nielsen RB, Norgaard M, Horsdal HT, Sturmer T, Larsen FB, Sorensen HT. Lifestyle profile among statin users. Epidemiology. 2013; 24:619-20. https:// doi.org/10.1097/EDE.0b013e318296e646. 